\title{
A Data-Driven Multiobjective Dynamic Robust Modeling and Operation Optimization for Continuous Annealing Production Process
}

\author{
Yao WANG, ${ }^{1)}$ Xianpeng WANG, ${ }^{21 *}$ Zhiming $\mathrm{DONG}^{21}$ and Zan WANG ${ }^{31}$ \\ 1) Key Laboratory of Data Analytics and Optimization for Smart Industry, Northeastern University, Ministry of Education, \\ Northeastern University, Shenyang, 110819 China. \\ 2) Liaoning Engineering Laboratory of Operation Analytics and Optimization for Smart Industry, Northeastern University, \\ Shenyang, 110819 China. \\ 3) Liaoning Key Laboratory of Manufacturing System and Logistics, Institute of Industrial \& Systems Engineering, \\ Northeastern University, Shenyang, 110819 China.
}

(Received on September 5, 2019; accepted on December 13, 2019; J-STAGE Advance published date: February 6, 2020)

\begin{abstract}
There are many dynamic disturbances during the continuous annealing production line (CAPL) in iron and steel enterprise. Traditional robust operation optimization considers only the maximum disturbance range in previous production and overrides the dynamic changes of these disturbances, which often results in high production cost and low product quality. Therefore, this paper proposes a novel multiobjective dynamic robust optimization (MODRO) modeling method by further taking into account the dynamic changes of these disturbances and adopting a time series prediction model based on a least square support vector regression (LSSVR) to predict the range of disturbances in next time slot. The main feature of the model is that the robustness can be dynamically adjusted according to the disturbance range predicted by the LSSVR. To solve this model, an improved NSGA-Il algorithm is developed based on a new crowding metric. Numerical results based on actual production process data illustrate that the proposed MODRO modeling method is obviously superior to traditional static robust operation optimization, and that it can significantly improve the strip quality and the capacity utilization of the CAPL, and reduce the total energy consumption.
\end{abstract}

KEY WORDS: continuous annealing; time series prediction; dynamic robust operation optimization; multiobjective evolutionary algorithm.

\section{Introduction}

The continuous annealing production line (CAPL) is shown in Fig. 1, in which the most important stages mainly consist of heating, soaking, slow cooling, rapid cooling, over-aging and quenching. During production, the strips are firstly welded one by one in the welding mill and then pickled to remove oxide scale. After that, each strip will be processed continuously through heating furnace (HF), soaking furnace (SF), slow cooling furnace (SCF), fast cooling furnace (FCF), over-aging furnace (OAF), and quenching furnace $(\mathrm{QF})$. Finally, the strip will be coiled after processed by the skin pass mill (SPM). The setting of control variables in stages from heating to skin pass mill directly determine the strip quality and energy consumption of CAPL.

The production process operation optimization is located between the production scheduling layer and the process control layer in the automation system of CAPL. Its task is

* Corresponding author: E-mail: wangxianpeng@ise.neu.edu.cn DOI: https://doi.org/10.2355/isijinternational.ISIJINT-2019-570 to achieve the optimal setting of process control variables with consideration of the production constraints so that the economic indicators such as strip quality, energy consumption, and capacity utilization can be optimized simultaneously.

There are many control variables in the CAPL and these variables are strongly coupling with each other. Besides, many dynamic disturbances exist during production. For example, the temperature of each furnace is measured by radiation temperature measurement, so the inadequate combustion of gas in the furnace will lead to inaccurate measurement of temperature. The dynamic disturbances of oxygen content in the furnace will also lead to changes in combustion efficiency of gas. The dynamic disturbances of controllers often cause temperature permutations of furnaces. These dynamic disturbances make the operation optimization of CAPL very difficult, and the traditional human-based method cannot achieve the global optimization of CAPL. For the operation optimization of CAPL, Guo et al. ${ }^{1)}$ established a mathematical model to minimize the total energy consumption and proposed a particle swarm 


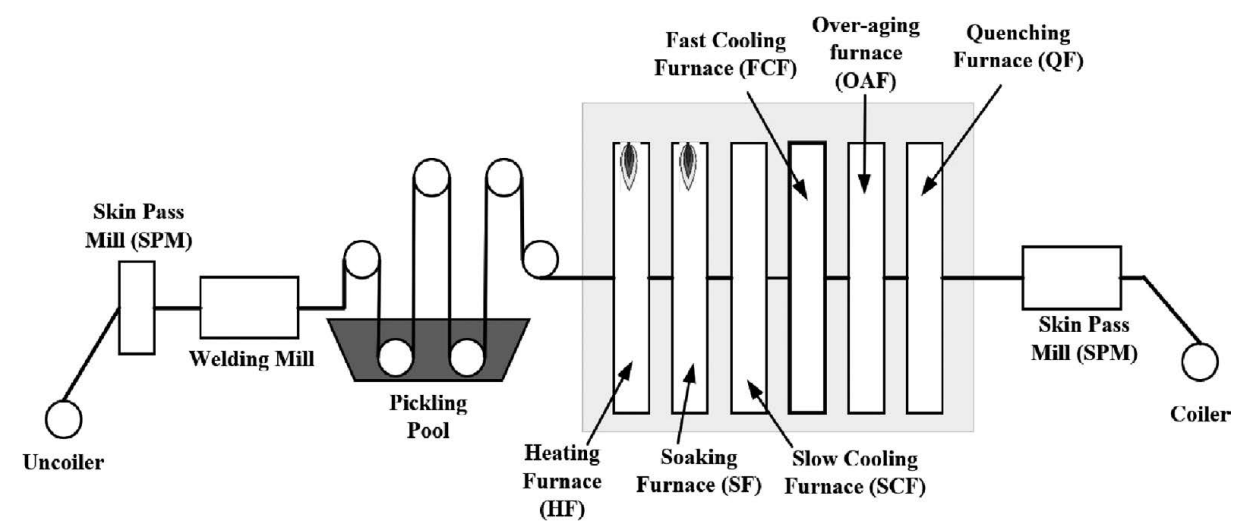

Fig. 1. Illustration of continuous annealing production process.

optimization algorithm to solve the model. However, this model did not take into account the dynamic disturbances in practical production. A data-driven model to predict the strip temperature in CAPL was proposed by Zhang et al., ${ }^{2)}$ which could help to improve the accuracy of the prediction results.

In recent years, there have been many researches focused on the operation optimization problems in process industries such as the iron and steel industry and the chemical industry. ${ }^{3-11)}$ For the gas stirred ladle systems in the steelmaking process, Mazumdar et al. ${ }^{3)}$ formulated a multiobjective optimization model with constraints to investigate inert gas injection in steelmaking ladles. In view of the hot stamping process, $\mathrm{Mu}$ et al. $^{4)}$ established the response surface model to optimize the heating parameters of hot stamping. For the blast furnace ironmaking process, Helle et al. ${ }^{5)}$ formulated the task of top gas recycling as a multiobjective optimization problem. Gao et al. ${ }^{6}$ ) and Wang et al. ${ }^{7)}$ respectively proposed a new parallel hybrid algorithm and a parallel differential evolution algorithm to solve the multiobjective operation optimization of the naphtha pyrolysis process. For the hot rolling production process, Jia et al. ${ }^{8)}$ proposed the optimal design framework by using multiobjective optimization to obtain the optimal hot rolling parameters. Chen et al. ${ }^{9)}$ established the operation optimization model of hot rolling process with the more practical constraints and presented a hybrid self-adaptive genetic algorithm to solve this model. Xia et al ${ }^{10,11)}$ developed a novel optimization method to solve the operation optimization problem of furnace temperature in the slab reheating process and proposed a mechanism and data analytics based hybrid model to study the furnace operation optimization problem. However, these research usually did not consider the dynamic disturbances, which meant that the production process was static.

To handle the dynamic disturbances or uncertainties in production process, robust operation optimization (ROO) has been widely used in recent years. ${ }^{12)}$ The main idea of ROO is to provide a relatively conservative solution that can optimize the objective function and at the same time ensure the stable and safe operation of the production process even when disturbances occur. For the uncertainties during the cold rolling production process, a ROO model with the goal of energy consumption minimization of the cold rolling mill was constructed by Hong and Wang. ${ }^{13)}$ Leiras et al. $^{14)}$ took into account the fluctuations of product price, cost, product output and demand, and proposed a ROO model for crude oil refining process with the objec- tive of maximizing the expected total benefit. Govatsmark and Skogestad ${ }^{15)}$ investigated a chemical process consisting of a reactor and a separator and proposed a ROO model to ensure process safety. Yasari et al. ${ }^{16)}$ established a multiobjective robust operation optimization (MOROO) model for the oil field production process, and adopted a multiobjective evolutionary algorithm based on NSGA-II to obtain the robust water injection operation schemes for each oil well. In these traditional ROO, the robustness of operation optimization is often calculated according to the maximum disturbance range using the Monte Carlo method. For example, if the maximum disturbance range of a certain control variable is $a \%$, the robustness is calculated by the formulation $\hat{f}(X)=\sum_{i=1}^{n} f\left(X+\varphi\left(\sigma_{i}\right)\right) / n$, where $X$ denotes the set of control variables, $f(X)$ is the objective value of $X$, $\sigma_{i}$ is the $i$ th sampling result of disturbance within the given range $a \%$ using the Monte Carlo method, and $\varphi\left(\sigma_{i}\right)$ is the corresponding change of control variables caused by the disturbance $\sigma_{i}$. That is, the average objective of $n$ solutions sampled by the Monte Carlo method within the given range $a \%$ is taken as the robustness. ${ }^{17)}$

Although ROO has been widely used to guarantee operation stability and safety, it is based on the assumption that the disturbances will not exceed the maximum value in all the production horizon. However, in practical production it cannot be guaranteed that this assumption can always be satisfied. That is, a certain significant disturbance exceeding the maximum value will make current solution infeasible and bring considerable risk to production safety. Besides, if the process is always operated under the traditional ROO framework, the total production cost will be high and the product quality may be deteriorated because the disturbance range may be small in most production time. Therefore, it is very necessary to further take into account the dynamic change of disturbances in ROO, which is called the dynamic robust operation optimization (DROO) problem in this paper. The main idea of DROO is to use the time series prediction method to predict the dynamic changes of disturbances in the next production cycle and then based on the prediction result adjust the extent of robustness of the solutions accordingly, so as to further improve the quality of operation optimization. Figure 2 compares of the traditional $\mathrm{ROO}$, in which the robustness is static because the disturbance range is fixed at the maximum value in the given rolling time horizon, and the proposed DROO, in which the given rolling time horizon is equally divided into $m$ time 


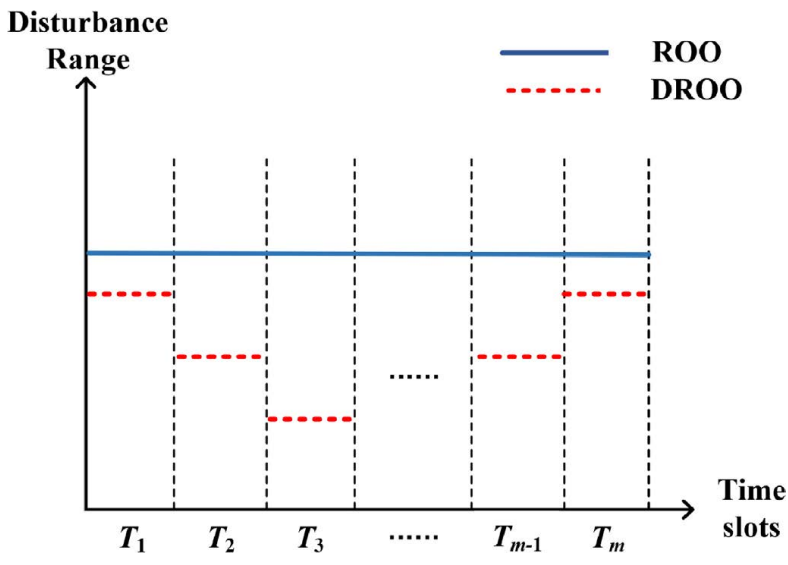

Fig. 2. Comparison of static ROO and DROO for defining the robustness. (Online version in color.)

slots and a time series prediction model based on the least square support vector regression (LSSVR) ${ }^{18)}$ is adopted to dynamically get the predicted maximum disturbance range for the next time slot. The major feature of the proposed DROO is that the robustness can be dynamically adjusted according to the change of disturbance range instead of a fixed value, which in turn can help to achieve more elaborate settings of control variables.

The rest of the paper is organized as follows. In Section 2, the multiobjective DROO model for the CAPL (denoted as MODROO-CAPL) is established. The solving strategy of the MODROO-CAPL model based on the improved NSGAII algorithm and the time series prediction model based on LSSVR is presented in Section 3. Experimental results are presented and discussed in section 4. Finally, Section 5 concludes the paper.

\section{Multiobjective Dynamic Robust Operation Optimi- zation for CAPL}

\subsection{Definition of Multiobjective Dynamic Robustness of a Solution (MODRS)}

Different from the traditional definition of solution robustness in $\left.\mathrm{ROO},{ }^{17}\right)$ the definition of MODRS based on the time series prediction of disturbance changes can be described as follows.

As shown in Fig. 2, the whole production time horizon is firstly divided into $m$ equal time slots, i.e., $T_{1}, T_{2}, \ldots, T_{m}$, and then the maximum disturbance range in each time slot is obtained by the time series prediction model based on LSSVR. Finally, based on the $n$ sampling results of disturbance in each time slot using the Monte Carlo method, the $k$ th objective of a solution in the MODROO-CAPL in time slot $T_{i}$ can be calculated by Eq. (1).

$$
\hat{f}_{k}(X)=\frac{1}{n} \sum_{j=1}^{n} f_{k}\left(X+\varphi\left(\delta_{i j}\right)\right)
$$

where $f_{k}(X)$ is the $k$ th objective value of $X$ with the mechanism model of CAPL, $\delta_{i j}$ is the $j$ th sampling result of disturbance within the predicted disturbance range by LSSVR in the time slot $T_{i}$ using the Monte Carlo method, and $\varphi\left(\delta_{i j}\right)$ is the corresponding change of control variables caused by the disturbance $\delta_{i j}$.

\subsection{Mathematical Model of MODROO-CAPL}

2.2.1. Variables and Parameters

As shown in Fig. 1, there are many consecutive production stages in the CAPL. The decision (or control) variables in this problem mainly include the temperature of each stage (i.e., $\mathrm{HF}, \mathrm{SF}, \mathrm{SCF}, \mathrm{FCF}, \mathrm{OA}$, and $\mathrm{QF}$ ), the passing speed of the strip, and the rolling force of the SPM. The detailed definitions of these decision variables are shown in Table 1.

Besides the above decision variables, there are some production parameters, which are strongly related to the strip quality, in production processes before the CAPL such as hot rolling and continuous descaling and cold rolling mill (CDCM). These production parameters shown in Table 2 will be used in the calculation of the first objective (i.e., strip quality) based on the LSSVR model, in which the variables and production parameters will be used as input features and the corresponding strip quality as the output.

\subsubsection{Mathematical Model in Each Time Slot}

Based on the above parameters and variables, as well as the definition of MODRS, the mathematical model of the MODROO-CAPL in each time slot $T_{i}(i=1,2, \ldots, m)$ can be established as follows.

$$
\begin{aligned}
& \min \hat{f}_{1}(X)=\frac{1}{n} \sum_{j=1}^{n} f_{1}\left(X+\varphi\left(\delta_{i j}\right)\right) \\
& =\frac{1}{n} \sum_{j=1}^{n}\left|Q\left(X+\varphi\left(\delta_{i j}\right)\right)-Q_{r e q}\right| \\
& \min \hat{f}_{2}(X)=\frac{1}{n} \sum_{j=1}^{n} f_{2}\left(X+\varphi\left(\delta_{i j}\right)\right) \\
& =\frac{1}{n} \sum_{j=1}^{n}\left(\sum_{l=2}^{8}\left(x_{l}+\varphi\left(\delta_{i j}\right)_{l}\right) / 7\right) \\
& \min \hat{f}_{3}(X)=\frac{1}{n} \sum_{j=1}^{n} f_{3}\left(X+\varphi\left(\delta_{i j}\right)\right)=\frac{1}{n} \sum_{j=1}^{n} \frac{1}{\left(x_{1}+\varphi\left(\delta_{i j}\right)_{1}\right)} \\
& L_{l} \leq x_{l} \leq U_{l}, l=1,2, \ldots, 21 \\
& x_{2} \leq x_{3} \leq x_{4} \leq x_{5} \leq x_{6}
\end{aligned}
$$

In the above model, there three objectives to be simultaneously optimized, namely the strip quality, the energy consumption, and the capacity utilization. The first objective in Eq. (2) is expressed by the minimization of the deviation between the practical quality (the strip hardness is taken as the quality index) obtained by the decision vector $X$ and the required quality denoted by $Q_{\text {req. }}$. Due to the fact that the precise mechanism model of CAPL cannot be obtained, we use the LSSVR modeling method to construct the data-driven prediction model of strip quality, i.e., $Q\left(X+\varphi\left(\delta_{i j}\right)\right)$. Since the LSSVR is quite similar to that used in the time series prediction for disturbance range, we only give the procedure of LSSVR for the prediction of disturbance range in Section 3. The second objective in Eq. (3) is used to minimize the total energy consumption in HF and SF, which is expressed by the minimization of average temperature of the seven zones in HF and SF. The third objective in Eq. (4) is expressed 
Table 1. Definitions of decision variables.

\begin{tabular}{cccccc}
\hline$x_{1}$ & passing speed of the strip & $x_{8}$ & temperature of zone 2 in SF & $x_{15}$ & water temperature in QF \\
$x_{2}$ & temperature of zone 1 in HF & $x_{9}$ & cooling gas temperature of zone 1 in SCF & $x_{16}$ & elongation in SPM \\
$x_{3}$ & temperature of zone 2 in HF & $x_{10}$ & cooling gas temperature of zone 2 in SCF & $x_{17}$ & rolling force of roller 1\# in SPM \\
$x_{4}$ & temperature of zone 3 in HF & $x_{11}$ & cooling gas temperature in FCF & $x_{18}$ & rolling force of roller 2\# in SPM \\
$x_{5}$ & temperature of zone 4 in HF & $x_{12}$ & temperature in OAF 1\# & $x_{19}$ & entrance tension of strip in SPM \\
$x_{6}$ & temperature of zone 5 in HF & $x_{13}$ & temperature of zone 1 in OAF 2\# & $x_{20}$ & middle tension of strip in SPM \\
$x_{7}$ & temperature of zone 1 in SF & $x_{14}$ & temperature of zone 2 in OAF 2\# & $x_{21}$ & outlet tension of strip in SPM \\
\hline
\end{tabular}

Table 2. Production parameters used in the calculation of the first objective.

\begin{tabular}{cccccc}
\hline$e_{1}$ & width of the strip & $e_{5}$ & phosphorus content in the strip & $e_{9}$ & outlet temperature of re-heating furnace in hot rolling \\
$e_{2}$ & thickness of the strip & $e_{6}$ & manganese content in the strip & $e_{10}$ & average finishing temperature in hot rolling \\
$e_{3}$ & carbon content in the strip & $e_{7}$ & nitrogen content in the strip & $e_{11}$ & average coiling temperature in hot rolling \\
$e_{4}$ & sulphur content in the strip & $e_{8}$ & silicon content in the strip & $e_{12}$ & strip elongation in CDCM \\
\hline
\end{tabular}

by the minimization of the reciprocal of the passing speed of the strip, which in turn helps to maximize the capacity utilization of CAPL because higher speed can bring higher product output. Please note that in Eqs. (3) and (4) we use $\varphi\left(\delta_{i j}\right)_{l}$ to denote the corresponding change of the $l$ th decision variable in $X$ caused by the disturbance $\delta_{i j}$. Constraints (5) give the feasible ranges for each decision variable in which $L_{i}$ and $U_{i}$ represent the lower bound and upper bound for decision variable $x_{i}$, respectively. Constraint (6) requires that the temperature of five zones in HF should be in a nondescending order.

In the above mathematical model, the objectives are conflicting with each other. For example, the decrease of temperature settings in $\mathrm{HF}$ and $\mathrm{SF}$ can help to improve the second objective, however, the strip passing speed must be decreased accordingly so as to guarantee the strip quality, which will inevitably result in the increase of the third objective. So, the dynamic robust operation optimization of CAPL is a multiobjective optimization problem. According to the Pareto concept, a solution $X_{1}$ is said to dominate another solution $X_{2}$ if and only if $\hat{f}_{k}\left(X_{1}\right) \leq \hat{f}_{k}\left(X_{2}\right)$ for $\forall k \in\{1,2, \ldots, K\}$ and $\exists k \in\{1,2, \ldots, K\}$ for which $\hat{f}_{k}\left(X_{1}\right)<\hat{f}_{k}\left(X_{2}\right)$. A solution $X^{*}$ is said to be Pareto optimal if and only if there exists no solution in the decision space that can dominate $X^{*}$. Then the set of all Pareto optimal solutions is called the Pareto set, and the corresponding set of Pareto optimal vectors in the objective space is called the Pareto front (PF).

\section{Solving Strategy of MODROO-CAPL Model}

\subsection{Time Series Prediction Model Based on LSSVR}

To obtain the training data from the given time series of process data in CAPL, we adopt the phase space reconstruction method, which takes the previous $K$ data as the input feature and the next $K+1$ data as the output. Based on the obtained training data, the LSSVR is adopted as the prediction model. To achieve a better setting of parameters of LSSVR, we take the parameter setting as an optimization problem whose objective is to minimize the root mean squared error of the LSSVR model and the self-adaptive genetic algorithm $(\mathrm{SaGA})^{8)}$ is adopted to solve the problem.
Due to page limitation, please refer to Suykens and Vandewalle ${ }^{18)}$ and Chen et al. ${ }^{9}$ for details.

\subsection{An Improved NSGA-II for MODROO-CAPL}

In recent years, many kinds of multiobjective evolutionary algorithms (MOEAs) have been proposed in the literature. ${ }^{19-29)}$ Among these MOEAs, NSGA-II is one of the most widely adopted algorithms for practical optimization problems for process operation, ${ }^{3,4,6,8-11,27-30)}$ due to its characteristics of fast computation speed and good convergence. Therefore, in this paper we prefer to adopt the NSGA-II as the solving method. However, based on the analysis of some limitation of traditional NSGA-II, we also propose an improved definition of crowding metric based on solution distribution and thus develop an improved NSGA-II algorithm.

\subsubsection{New Crowding Distance Metric Based on Solution Distribution}

The main idea of generating the new population for the next generation $\left(P_{\text {new }}\right)$ in NSGA-II can be briefly described as follows. Firstly, the solutions of the original population $P$ are merged with all the new solutions to obtain $2 N$ solutions. Secondly, the fast non-dominated sorting approach is used to sort these solutions into different non-dominated fronts. Subsequently, $P_{\text {new }}$ can be established as follows. Starting from the first nondominated front, if the size of $P_{\text {new }}$ after addition of the solutions in this front into $P_{\text {new }}$ does not exceed $N$, the solutions of this front are added into $P_{\text {new }}$ and then the next front is considered and similar process will be repeated. Otherwise, the solutions in current front will be sorted according to the crowding distance and the most crowded solutions will be removed until the size of $P_{\text {new }}$ after the addition of solutions in current front reaches $N$. That is, the crowding distance is only applied to the selection of solutions in the last front to be added into $P_{n e w}$. Obviously, this method only considers the diversity of solutions in the last front, but does not take into account the diversity (or crowding) of solutions in adjacent fronts. Therefore, the selection method based on current crowding distance metric cannot guarantee the good distribution of selected solutions in $P_{\text {new }}$. 
In view of this situation, we propose a new metric for defining the crowding distance of a solution. The main idea is that the crowding distance of a solution should depend on the distribution difference between it and the solutions already added in the new population $P_{\text {new }}$ in the objective space, and that this solution can be added into $P_{\text {new }}$ only if it is quite different in the distribution from those solutions already in $P_{\text {new }}$. According to this idea, the new crowding distance metric of a solution $X$ can be defined by the minimum angle between this solution and any solution in $P_{\text {new }}$, as shown in Eq. (15) and Fig. 3(a). It can be seen that the smaller the value is, the more crowded the solution is. The reason is that a very small value of the metric means that there has been at least one solution that is better than or similar to the current solution in $P_{n e w}$. This metric can show the diversity of solutions between different fronts, instead of only a single front in NSGA-II.

$$
C\left(X, P_{\text {new }}\right)=\min \left\{\arccos \frac{F(X) \cdot F(Y)}{\|F(X)\| \times\|F(Y)\|}, \forall y \in P_{\text {new }}\right\} \ldots
$$

To illustrate the difference between the traditional crowding distance of NSGA-II and the proposed crowding distance metric based on solution distribution, an example is given based on Fig. 3(a). In this example, there are a total of 17 candidate solutions that are separated into 2 Pareto fronts and the population size is $N=13$. Since $\left|P F_{1}\right|=10$, all solutions in $P F_{1}$ will be added to $P_{\text {new }}$. For solutions in the second front, the traditional crowding distance of NSGA-II will firstly add the two extreme solutions $\left(X_{1}\right.$ and $\left.X_{7}\right)$ and then another solution $\left(X_{6}\right)$ with the largest crowding distance value into $P_{\text {new }}$ (Fig. 3(b)). However, the proposed crowding distance metric based on solution distribution will only add the three solutions $X_{3}, X_{4}$ and $X_{5}$ into $P_{n e w}$ because their crowding distances calculated by the new metric are larger than the other solutions in the second front (Fig. 3(c)). From Fig. 3(b), it can be seen that there exists a big area in the objective space that is not covered by $P_{n e w}$, which means that the diversity of $P_{\text {new }}$ is not good enough. On the contrary, it is clear from Fig. 3(c) that the three solutions $X_{3}, X_{4}$ and $X_{5}$ can be maintained in $P_{\text {new }}$ with help of the proposed new crowding distance metric.

\subsubsection{New Population Construction Method Based on the New Crowding Distance Metric}

Based on the above new definition of crowding distance metric, the construction method of new population can be presented in Algorithm 1 (shown in Fig. 4). There are two main differences between this construction method and that in NSGA-II: first, the new crowding distance metric will be used in the solution selection from the second front to the last possible front (lines 8-19) because it can evaluate the diversity between the candidate solutions and all solutions that have been added into $P_{\text {new }}$, instead of only the last possible front in traditional NSGA-II; second, a threshold value of crowding distance metric is used in the solution selection to improve the diversity of new population. That is, only the solution whose minimum angle is greater than

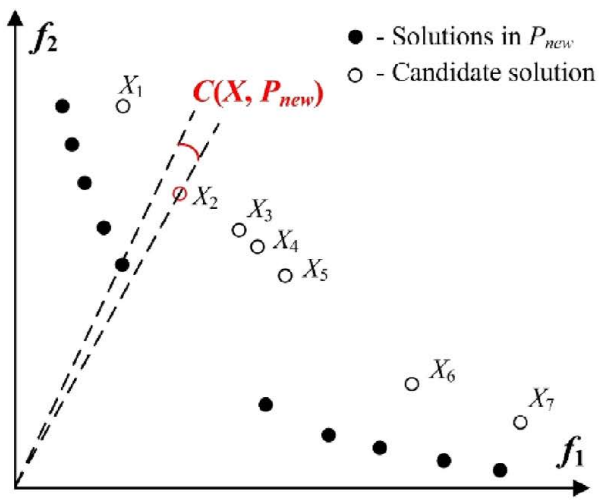

(a) Illustration of definition of the crowding distance based on solution distribution

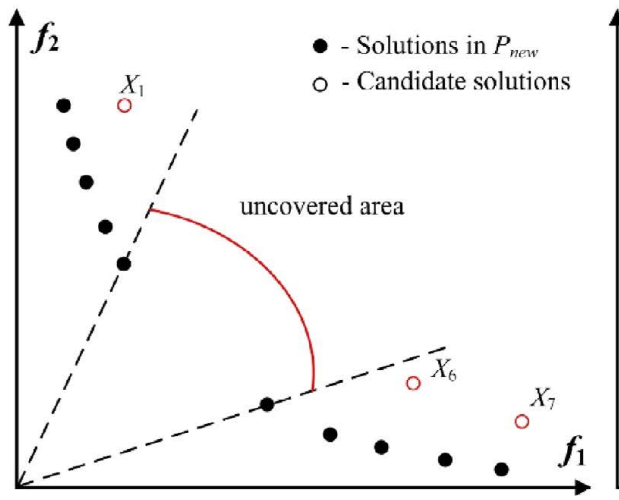

(b) $P_{\text {new }}$ obtained by traditional crowding distance metric

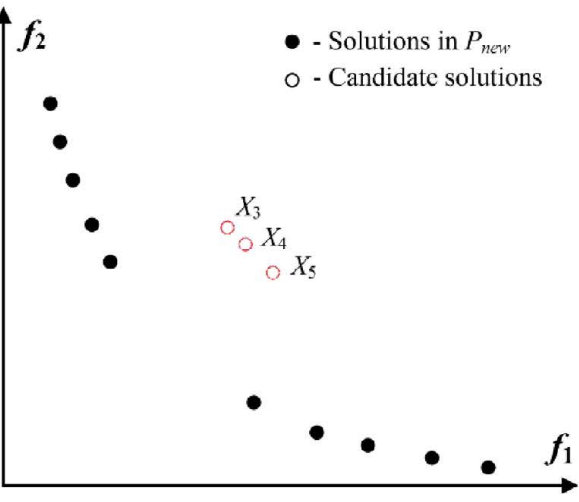

(c) $P_{\text {new }}$ obtained by new crowding distance metric based on solution distribution

Fig. 3. Comparison between traditional crowding distance metric and the proposed crowding distance metric. (Online version in color.) 


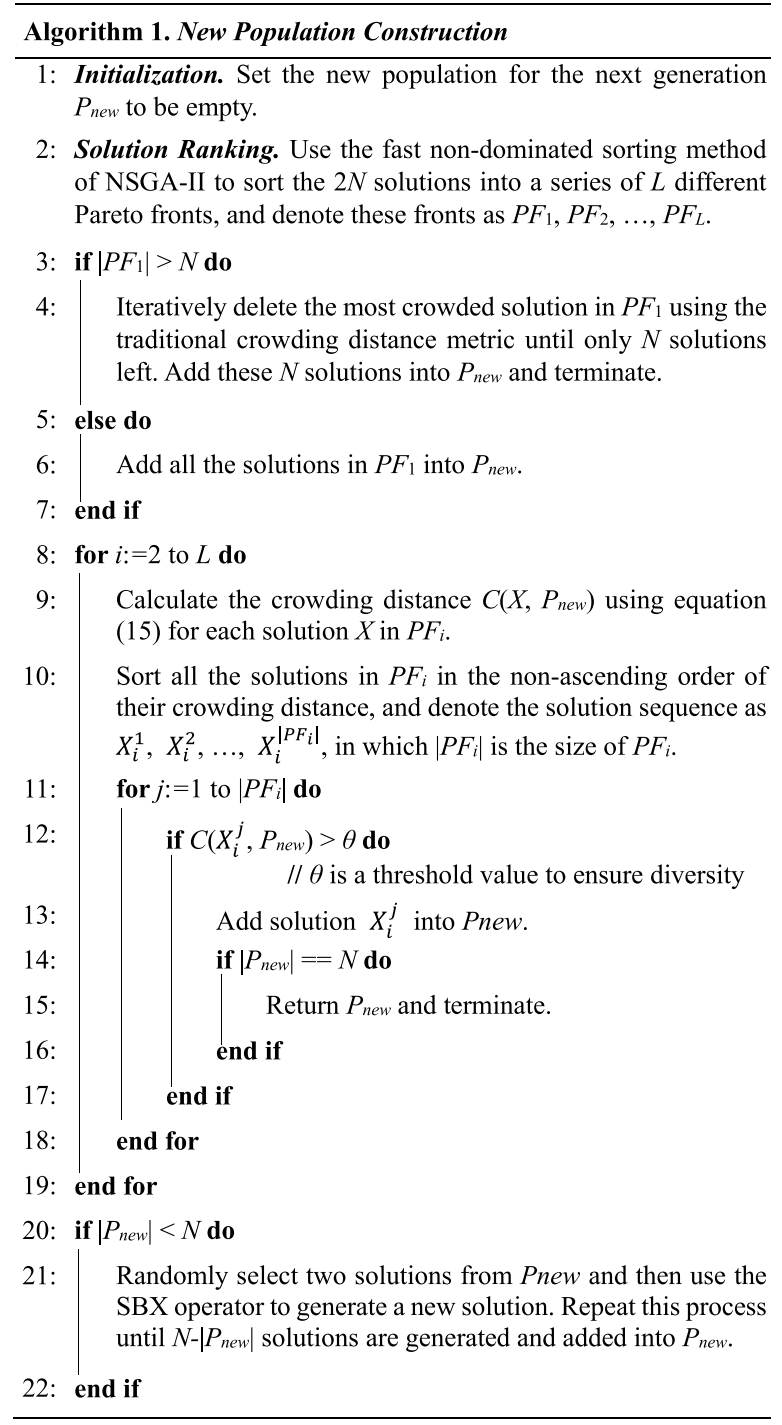

Fig. 4. Generation of new population based on the new crowding metric.

the threshold value $\theta$ (line 12) can be added to $P_{\text {new }}$. So the number of solutions in $P_{\text {new }}$ after the solution selection of the final front may be less than $N$, and to handle this situation, the $S B X$ operator $^{20)}$ is employed to randomly generate new solutions for $P_{\text {new }}$ (lines 20-22), which in turn can also help to improve the diversity of $P_{\text {new }}$.

\subsubsection{Procedure of the Improved NSGA-II}

Based on the above sections, the detailed procedure of the improved NSGA-II algorithm can be presented in Algorithm 2 (Fig. 5), in which an external archive EXA is used to store the best non-dominated solutions obtained by the algorithm.

\section{Numerical Experiments}

To verify the performance of the MODROO-CAPL model and the improved NSGA-II algorithm proposed in this paper, extensive numerical experiments are carried out in this section. Firstly, the validity and accuracy of the time series prediction model (TSPM) based on LSSVR is verified on the standard training data in subsection 4.1, and four functions are constructed to simulate the relative disturbance variation of the control variables. Then, we carry out the

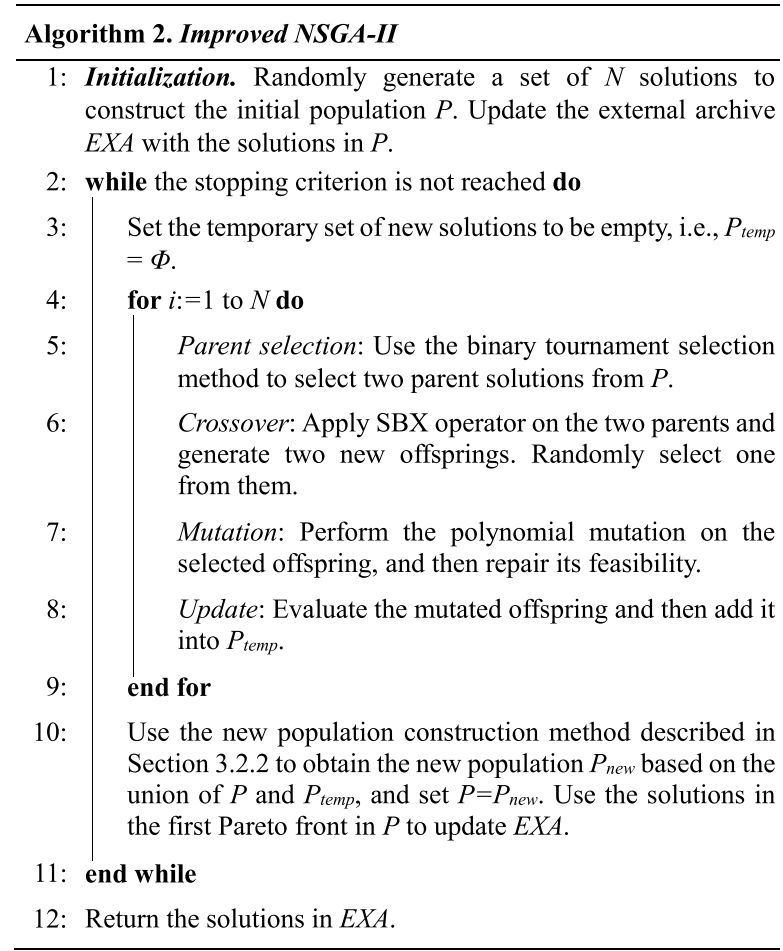

Fig. 5. Procedure of the improved NSGA-II.

comparative studies between our improved NSGA-II and four start-of-the-art MOEAs in subsection 4.2. In Subsection 4.3 we compare our MODROO-CAPL model with the traditional multiobjective ROO (MOROO) model for CAPL.

All the experiments are carried out on a personal computer with the Intel Core $17-7700 \mathrm{~K} 4.20 \mathrm{GHz}$ CPU, 16.0 GB memory, and Windows 10.0 64-bit operating system.

\subsection{Performance Analysis of TSPM Based on LSSVR}

In order to verify the effect of the TSPM based on LSSVR, the time series prediction is performed on the Darwin sea level pressures data from UCI database. A total of 400 training samples are obtained by using the phase space reconstruction, and 30 times of single-step prediction are carried out as the testing data. The experimental results are shown in Table 3 and Fig. 6, in which the root mean squared error (RMSE) and average relative error (ARE) are used as the evaluation metrics.

As can be seen from Table 3 and Fig. 6, the TSPM based on LSSVR has a good performance. In the actual production process, the disturbance of the control variable may make the variable larger (i.e., the disturbance fluctuation value is positive) or smaller (i.e., the disturbance fluctuation value is negative). The absolute value of the disturbance fluctuation value that makes the biggest change of each control variable in each time slot is selected as the maximum disturbance range value of this variable in this slot. As shown in Fig. 7, four functions are constructed to simulate the relative disturbances of control variables. The four functions have different frequency and different forms of variation, and noise is added to each function to make it close to the actual production situation. The TSPM based on LSSVR is performed on the four simulated functions. A total of 400 training samples are obtained by using the phase space reconstruction, and 30 times of single-step prediction are 
carried out as the testing data.

From the experimental results shown in Table 4 and Fig. 7 for the 30 testing data samples, we can know that the prediction accuracy of the four simulation disturbance functions is relatively good, and the gap between the predictive value and the actual value in the actual production is comparative small, which has little influence on the results in the simulation of the actual production process and meets the actual production needs.

Table 3. Experimental results on the Darwin sea level pressures data.

\begin{tabular}{ccc}
\hline & Training data result & Testing data result \\
\hline RMSE & 0.568 & 0.934 \\
ARE & $5.07 \%$ & $7.63 \%$ \\
\hline
\end{tabular}

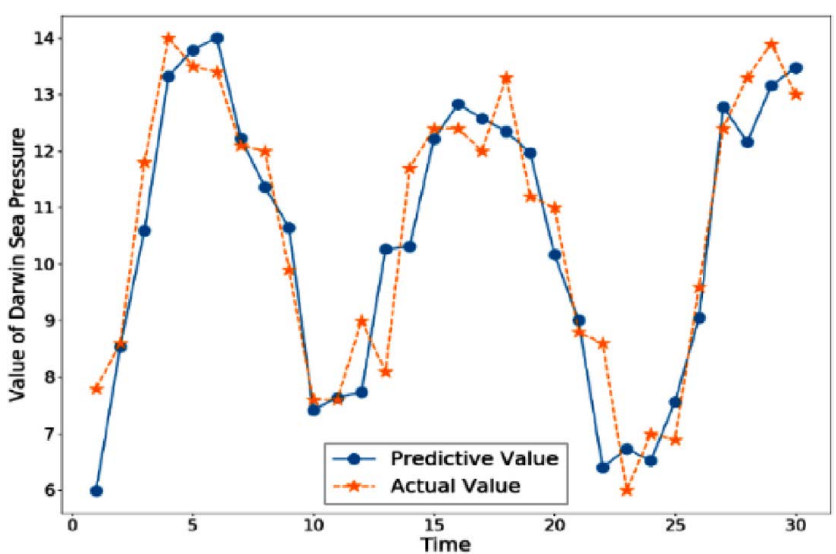

Fig. 6. The actual values and predicted values of 30 times of single-step prediction for the testing data. (Online version in color.)

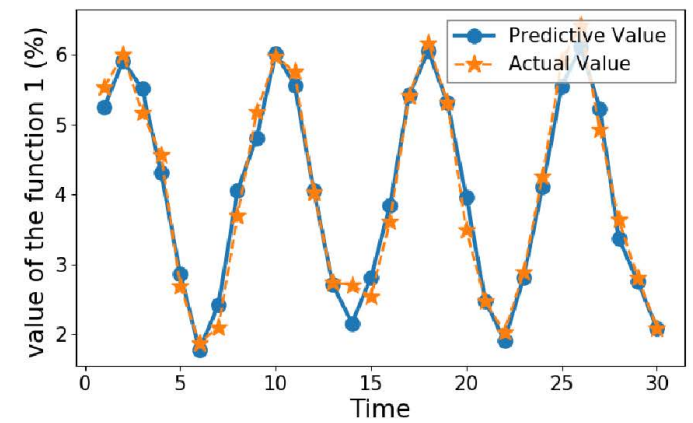

(a) the result of Simulated function 1

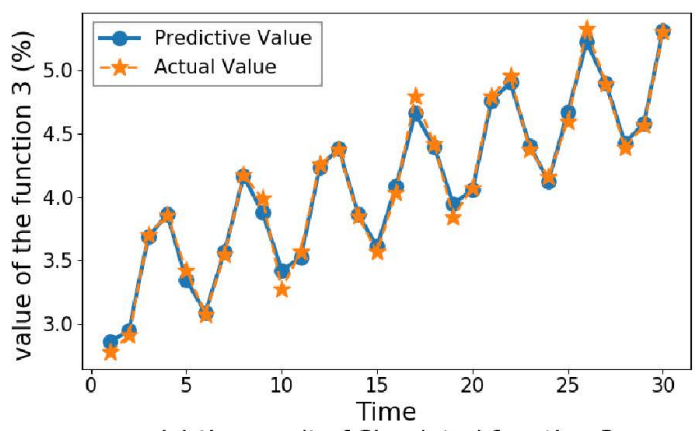

(c) the result of Simulated function 3

\subsection{Performance Analysis of Improved NSGA-II on Benchmark Problems}

To verity the performance of the improved NSGA-II proposed in Section 3.2, we carry out the comparative studies between our improved NSGA-II and four start-of-the-art MOEAs in the literature on the two series of benchmark problems, i.e., $\mathrm{ZDT}^{31)}$ and CEC2009. ${ }^{32)}$

In this paper, we choose the inverted generational distance (IGD) ${ }^{33)}$ as our performance metric. IGD can comprehensively consider the diversity and convergence properties of a set of non-dominated solutions, so it is widely used in evaluating the performance of multiobjective algorithms. The smaller the IGD value, the higher the quality and diversity of the set of nondominated solutions will have.

The parameter settings of the improved NSGA-II algorithm are as follows: the population size is set to 100 , the cross probability and mutation probability are set to be the same with traditional NSGA-II, i.e., $P_{c}=0.95$ and $P_{m}=1 / D(D$ is the number of variables), and the minimum angle threshold $\theta=1^{\circ}$. The stopping criterion is set to be the maximum function evaluations, which is set to 30000 for the bi-objective problems and 50000 for the tri-objective problems.

We compare the improved NSGA-II proposed with four state-of-the-art MOEAs, i.e., traditional NSGA-II, ${ }^{19}$ GDE3 $^{34)}$ MOEA/D-STM ${ }^{26)}$ and AbYSS. $^{21)}$ These algo-

Table 4. Experimental results of the 30 testing data on four simulated functions.

\begin{tabular}{ccc}
\hline Function & RMSE of testing data & ARE of testing data \\
\hline 1 & $2.47 \mathrm{e}-1$ & $5.36 \%$ \\
2 & $3.08 \mathrm{e}-1$ & $5.97 \%$ \\
3 & $6.10 \mathrm{e}-2$ & $1.20 \%$ \\
4 & $9.20 \mathrm{e}-2$ & $1.27 \%$ \\
\hline
\end{tabular}

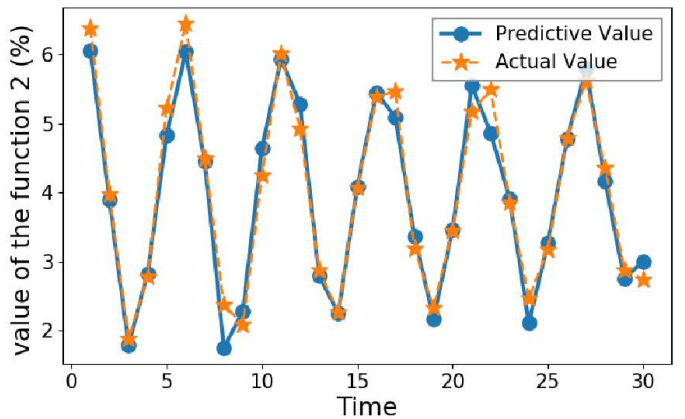

(b) the result of Simulated function 2

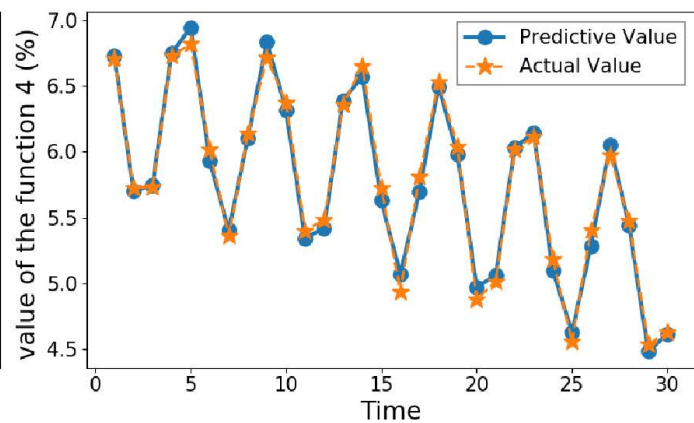

(d) the result of Simulated function 4

Fig. 7. The actual values and predictive values of 30 times of signal-step prediction for the four simulated functions. (Online version in color.) 
rithms were implemented by Java in the jMetal 5.0 software package that can be obtained from the website http://jmetal. sourceforge.net/. The parameter settings of these algorithms are set to the recommended values given in the corresponding literature.

For each test problem, each algorithm runs 30 independent times, and the statistical results of IGD are used for comparison. The Wilcoxon rank sum test is employed to compare the improved NSGA-II with the other four algorithms at a significance level of 5\%. The symbol "+" means that the improved NSGA-II is significantly better than its rival, while the symbol "_" means that the improved NSGA-II is significantly worse than its rival. Besides, the symbol "=" means that there is no significant difference between the algorithms. The best result for each problem is highlighted in gray background in the table. The mean value (denoted by Mean) of the 30 runs and the corresponding standard deviation (denoted by St.d.) are listed in Table $\mathbf{5}$.

In Table $5, N_{\text {better }}$ in the last row means the number of problems for which each algorithm achieves the best result. It can be seen in Table 5 that the improved NSGA-II algorithm can obtain the best results of mean and standard deviation metrics for 11 problems and that the MOEA/D-STM gets the best results for the left 4 test problems. Please note that if two algorithms achieve the same mean IGD, the one with a smaller St.d. value is considered to be better. More specifically, the improved NSGA-II is significantly better than both the GDE3 and traditional NSGA-II for all the 15 test problems, which illustrates that the proposed algorithm can achieve great improvement in the diversity and quality of the population. With comparison to MOEA/D-STM, the improved NSGA-II can obtain significantly better results for 11 out of the 15 test problems, and the MOEA/D-STM shows significantly better performance than that of the improved NSGA-II algorithm for 3 test problems. Besides, the improved NSGA-II achieves significantly better results than the AbYSS algorithm for 13 problems and for the first two problems there is no significant performance difference between the two algorithms. Based on these results, it can be concluded that the improved NSGA-II algorithm proposed in this paper is more robust for different problems and superior to the four powerful MOEAs in the literature.

\subsection{Performance Analysis of Improved NSGA-II on MODROO-CAPL}

\subsubsection{Experiment Setting}

Due to the fact that the true Pareto front of MODROCAPL is not known, in the experiment we employ the improved NSGA-II to solve the operation optimization problem of CAPL without consideration of distribution and take the obtained Pareto front as the referenced Pareto front to calculate IGD. Please note that the stopping criterion of the improved NSGA-II to get the referenced Pareto front is set to be a total of $10^{6}$ function evaluations. Since the consideration of disturbance will inevitably deteriorate the quality of the obtained Pareto front, it is reasonable to take the Pareto front of the operation optimization of CAPL without consideration of distribution as the referenced Pareto front to calculate IGD metric. Moreover, we will normalize the referenced Pareto front and the Pareto fronts obtained by the test algorithms before the calculation of IGD because the three objectives have different units and scales.

In the experiment, three cases of disturbance are designed to simulate the actual production process, namely single variable disturbance, bi-variable disturbance, and multivariable disturbance. The MODROO model and the traditional MOROO model are compared based on 6 steel strips of CAPL (please note that each strip means a problem), and for each strip the production time horizon is equally divided into 10 time slots. To collect the statistical results of IGD metric, a total of 15 independent runs are carried out for each strip.

Table 5. Comparison results of IGD for the improved NSGA-II and four other powerful MOEAs.

\begin{tabular}{|c|c|c|c|c|c|c|c|c|c|c|}
\hline \multirow{2}{*}{ Problem } & \multicolumn{2}{|c|}{ GDE3 } & \multicolumn{2}{|c|}{ MOEA/D-STM } & \multicolumn{2}{|c|}{ AbYSS } & \multicolumn{2}{|c|}{ NSGA-II } & \multicolumn{2}{|c|}{ Improved NSGA-II } \\
\hline & Mean & St.d. & Mean & St.d. & Mean & St.d. & Mean & St.d. & Mean & St.d. \\
\hline ZDT1 & $4.55 \mathrm{e}-03^{+}$ & $3.66 \mathrm{e}-08$ & $5.80 \mathrm{e}-01^{+}$ & $1.52 \mathrm{e}-02$ & $3.85 e-03=$ & $2.17 \mathrm{e}-08$ & $4.86 \mathrm{e}-03^{+}$ & $3.22 \mathrm{e}-08$ & $3.81 \mathrm{e}-03$ & $9.09 \mathrm{e}-10$ \\
\hline ZDT2 & $4.67 \mathrm{e}-03^{+}$ & $3.12 \mathrm{e}-08$ & $1.06 \mathrm{e}+00^{+}$ & $5.70 \mathrm{e}-02$ & $3.92 \mathrm{e}-03=$ & $3.15 \mathrm{e}-09$ & $4.89 \mathrm{e}-03^{+}$ & $2.65 \mathrm{e}-08$ & $3.92 \mathrm{e}-03$ & $1.38 \mathrm{e}-09$ \\
\hline ZDT3 & $5.13 \mathrm{e}-03^{+}$ & $3.84 \mathrm{e}-08$ & $4.78 \mathrm{e}-01^{+}$ & $4.48 \mathrm{e}-03$ & $1.56 \mathrm{e}-02^{+}$ & $6.54 \mathrm{e}-04$ & $5.40 \mathrm{e}-03^{+}$ & $4.28 \mathrm{e}-08$ & $4.44 \mathrm{e}-03$ & $3.26 \mathrm{e}-09$ \\
\hline ZDT4 & $2.62 \mathrm{e}+00^{+}$ & $5.82 \mathrm{e}-01$ & $3.57 \mathrm{e}+00^{+}$ & $1.84 \mathrm{e}+00$ & $6.56 \mathrm{e}-03^{+}$ & $9.38 \mathrm{e}-06$ & $5.7 \mathrm{e}-03^{+}$ & $1.08 \mathrm{e}-06$ & $3.78 \mathrm{e}-03$ & $6.71 \mathrm{e}-10$ \\
\hline ZDT6 & $4.88 \mathrm{e}-03^{+}$ & $8.86 \mathrm{e}-0$ & $2.08 \mathrm{e}-01^{+}$ & $1.26 \mathrm{e}-0$ & $3.18 \mathrm{e}-03^{+}$ & 1. & $4.14 \mathrm{e}-03^{+}$ & 1. & $2.47 \mathrm{e}-03$ & 8. \\
\hline CEC2009_UF1 & $8.66 \mathrm{e}-02^{+}$ & $1.25 \mathrm{e}-04$ & $6.63 e-02^{-}$ & $1.53 \mathrm{e}-04$ & $1.65 \mathrm{e}-01^{+}$ & $2.73 \mathrm{e}-03$ & $1.16 \mathrm{e}-01^{+}$ & $8.89 \mathrm{e}-04$ & $7.70 \mathrm{e}-02$ & $4.02 \mathrm{e}-04$ \\
\hline CEC2009_UF2 & $5.27 \mathrm{e}-02^{+}$ & $6.45 \mathrm{e}-06$ & $2.98 \mathrm{e}-02^{-}$ & $1.73 \mathrm{e}-05$ & $6.89 \mathrm{e}-02^{+}$ & $1.12 \mathrm{e}-03$ & $4.47 \mathrm{e}-02^{+}$ & $1.79 \mathrm{e}-04$ & $3.72 \mathrm{e}-02$ & $9.02 \mathrm{e}-05$ \\
\hline CEC2009_UF3 & $3.81 \mathrm{e}-01^{+}$ & $1.87 \mathrm{e}-04$ & $2.03 \mathrm{e}-01^{+}$ & $9.20 \mathrm{e}-04$ & $2.82 \mathrm{e}-01^{+}$ & $3.74 \mathrm{e}-03$ & $1.97 \mathrm{e}-01^{+}$ & $9.80 \mathrm{e}-04$ & $8.54 \mathrm{e}-02$ & $1.69 \mathrm{e}-04$ \\
\hline CEC2009_UF4 & $5.18 \mathrm{e}-02^{+}$ & $4.69 \mathrm{e}-06$ & $7.95 \mathrm{e}-02^{+}$ & $2.82 \mathrm{e}-05$ & $6.97 \mathrm{e}-02^{+}$ & $1.18 \mathrm{e}-05$ & $5.78 \mathrm{e}-02^{+}$ & $3.57 \mathrm{e}-06$ & $4.63 e-02$ & $6.30 \mathrm{e}-07$ \\
\hline CEC2009_UF5 & $3.94 \mathrm{e}-01^{+}$ & $3.61 \mathrm{e}-02$ & $9.14 \mathrm{e}-01^{+}$ & $1.94 \mathrm{e}-02$ & $6.66 \mathrm{e}-01^{+}$ & $2.38 \mathrm{e}-02$ & $4.49 \mathrm{e}-01^{+}$ & $2.53 \mathrm{e}-02$ & $2.22 \mathrm{e}-01$ & $3.92 \mathrm{e}-03$ \\
\hline CEC2009_UF6 & $1.90 \mathrm{e}-01^{+}$ & $6.00 \mathrm{e}-03$ & $4.70 \mathrm{e}-01^{+}$ & $2.01 \mathrm{e}-03$ & $4.07 \mathrm{e}-01^{+}$ & $1.50 \mathrm{e}-02$ & $2.49 \mathrm{e}-01^{+}$ & $9.15 \mathrm{e}-03$ & $1.34 \mathrm{e}-01$ & $5.65 \mathrm{e}-03$ \\
\hline CEC2009_UF7 & $4.70 \mathrm{e}-02^{+}$ & $5.59 \mathrm{e}-06$ & $4.60 \mathrm{e}-02=$ & $9.27 \mathrm{e}-05$ & $3.10 \mathrm{e}-01^{+}$ & $1.56 \mathrm{e}-02$ & $1.52 \mathrm{e}-01^{+}$ & $1.50 \mathrm{e}-02$ & $5.27 \mathrm{e}-02$ & $3.53 \mathrm{e}-03$ \\
\hline CEC2009_UF8 & $2.03 \mathrm{e}-01^{+}$ & $8.17 \mathrm{e}-05$ & $1.45 \mathrm{e}-01^{-}$ & $5.69 \mathrm{e}-04$ & $2.59 \mathrm{e}-01^{+}$ & $5.78 \mathrm{e}-03$ & $2.23 \mathrm{e}-01^{+}$ & $1.66 \mathrm{e}-03$ & $1.67 \mathrm{e}-01$ & $2.50 \mathrm{e}-03$ \\
\hline CEC2009_UF9 & $2.53 \mathrm{e}-01^{+}$ & $4.21 \mathrm{e}-04$ & $1.63 \mathrm{e}-01^{+}$ & $7.44 \mathrm{e}-04$ & $3.84 \mathrm{e}-01^{+}$ & $1.37 \mathrm{e}-02$ & $3.11 \mathrm{e}-01^{+}$ & $3.03 \mathrm{e}-03$ & $1.11 \mathrm{e}-01$ & $1.00 \mathrm{e}-04$ \\
\hline CEC2009_UF10 & $2.35 \mathrm{e}+00^{+}$ & $3.62 \mathrm{e}-02$ & $1.49 \mathrm{e}+00^{+}$ & $2.12 \mathrm{e}-02$ & $6.50 \mathrm{e}-01^{+}$ & $1.22 \mathrm{e}-01$ & $4.26 \mathrm{e}-01^{+}$ & $8.58 \mathrm{e}-03$ & $3.21 \mathrm{e}-01$ & $3.05 \mathrm{e}-03$ \\
\hline$N_{\text {better }}$ & 0 & & $T$ & & 0 & & 0 & & 11 & \\
\hline
\end{tabular}


The four simulated disturbance functions (as shown in Fig. 7) are used to simulate the disturbance changes of the variables in the production process, and Table 6 shows the partial actual value and predicted value of maximum disturbance range for the four control variables in each time slot. For example, the value of 3.277 in the top-left corner means that the maximum disturbance range of Variable 1 in the first time slot is $3.277 \%$, which is used in the sampling of the Monte Carlo method. In the MODROO model, we use the LSSVR models established in Section 4.1 for the four functions to predict the disturbance range in each time slot and the prediction result of each time slot is used in the MODROO model. Finally, we compare the results obtained by the two methods to show the effectiveness and superiority of the MODROO model.

In terms of parameter settings, the sampling times of each time slot is set to $n=50$, the number of sampling points in MOROO in each time slot is also set to 50 , and the maximum function evaluations is set to 20000 . The other parameter settings are as same as those in Subsection 4.2. The Wilcoxon rank sum test is also employed to compare the MODROO with the MOROO at a significance level of $5 \%$. Please note that in both MODROO and MOROO the improved NSGA-II is adopted as the solver.

\subsubsection{Case 1: Single-variable Disturbance Change}

For the case that only one variable has the disturbance change in the actual production process, we select the elongation of SPM (i.e., $x_{16}$ ) as the variable whose change of disturbance range is simulated with Function 1 in Fig. 7. As described above, the time series prediction based on LSSVR is used in the MODROO model to predict the maximum disturbance range in each time slot, while in the traditional MOROO model the maximum disturbance range (i.e., 8.0\%) is used in the whole production time horizon (i.e., the maximum disturbance range is the same one for all time slots).

The comparison results between the two models are presented in Table 7. From the table, it is clear that the MODROO model can achieve better results than the traditional MOROO model for all time slots of each strip. For each strip, the IGD results of MODROO model is also significantly better than the IGDs obtained by the MOROO model for most time slots.

\subsubsection{Case2: bi-variable Disturbance Change}

For the case that two variables have the disturbance change in the actual production process, we select the elongation of SPM $\left(x_{16}\right)$ and the temperature of zone 3 in HF $\left(x_{4}\right)$ as the variables whose changes of disturbance range are simulated with Function 1 and Function 2 in Fig. 7, respectively. In the traditional MOROO model the maximum disturbance ranges of $8.0 \%$ and $8.0 \%$ are used in all time slots for $x_{16}$ and $x_{4}$, respectively.

The comparison results between the two models are presented in Table 8. Based on these comparison results, similar conclusions can be obtained, i.e., the MODROO model can achieve better results than the traditional MOROO model for all time slots of each strip and on average the MODROO model is superior to the traditional MOROO model for the case that the disturbance range changes for two variables.

\subsubsection{Case3: Multi-variable Disturbance Change}

For the case that multiple variables have the disturbance change in the actual production process, we select the elongation of SPM $\left(x_{16}\right)$, the temperature of zone 3 in HF $\left(x_{4}\right)$, the temperature of zone 1 in SF $\left(x_{7}\right)$, and the passing speed of the strip $\left(x_{1}\right)$ as the variables. Their changes of disturbance range are simulated with Function 1, Function 2, Function 3, and Function 4 in Fig. 7, respectively. In the traditional MOROO model the maximum disturbance ranges of $8.0 \%, 8.0 \%, 6.0 \%$ and $8.0 \%$ are used in all time slots for $x_{16}, x_{4}, x_{7}$ and $x_{1}$, respectively.

The comparison results between the two models are presented in Table 9. From the comparison results, it can be seen that the MODROO can get better results for all time slots, and that for the 55 out of 60 time slots the performance of MODROO is significantly better than the MOROO model.

\subsubsection{Results Analysis of Three Cases}

The comparison results between MODROO and tradi-

Table 6. Actual value of the maximum disturbance range of each variable in 10 time slots

\begin{tabular}{ccccccccc}
\hline & \multicolumn{7}{c}{ Actual value of maximum disturbance range (\%) } \\
\cline { 2 - 8 } Time Slot & \multicolumn{2}{c}{ Variable 1 } & \multicolumn{2}{c}{ Variable 2 } & \multicolumn{2}{c}{ Variable 3 } & \multicolumn{2}{c}{ Variable 4} \\
\cline { 2 - 9 } & $\begin{array}{c}\text { Actual } \\
\text { value }\end{array}$ & $\begin{array}{c}\text { Predicted } \\
\text { value }\end{array}$ & $\begin{array}{c}\text { Actual } \\
\text { value }\end{array}$ & $\begin{array}{c}\text { Predicted } \\
\text { value }\end{array}$ & $\begin{array}{c}\text { Actual } \\
\text { value }\end{array}$ & $\begin{array}{c}\text { Predicted } \\
\text { value }\end{array}$ & $\begin{array}{c}\text { Actual } \\
\text { value }\end{array}$ & $\begin{array}{c}\text { Predicted } \\
\text { value }\end{array}$ \\
\hline 1 & 3.277 & 3.570 & 4.900 & 5.133 & 2.810 & 2.830 & 5.715 & 5.643 \\
2 & 5.823 & 5.527 & 5.422 & 5.375 & 2.927 & 2.883 & 5.708 & 5.731 \\
3 & 4.968 & 5.252 & 3.790 & 4.088 & 3.670 & 3.659 & 6.765 & 6.786 \\
4 & 2.316 & 2.865 & 2.568 & 2.697 & 3.889 & 3.945 & 6.869 & 6.983 \\
5 & 2.235 & 2.285 & 3.158 & 3.051 & 3.380 & 3.413 & 6.026 & 5.997 \\
6 & 5.140 & 5.567 & 4.789 & 4.980 & 2.985 & 2.998 & 5.410 & 5.354 \\
7 & 5.899 & 6.343 & 5.550 & 5.315 & 3.599 & 3.516 & 6.124 & 5.999 \\
8 & 3.722 & 3.524 & 4.258 & 4.283 & 4.039 & 4.195 & 6.857 & 6.899 \\
9 & 1.872 & 2.119 & 2.827 & 2.776 & 3.840 & 3.922 & 6.370 & 6.370 \\
10 & 3.603 & 3.242 & 2.828 & 3.069 & 3.426 & 3.351 & 5.345 & 5.391 \\
\hline
\end{tabular}


ISIJ International, Vol. 60 (2020), No. 6

Table 7. Computational results of IGD for the first three strips obtained by MOROO and MODROO for Case 1

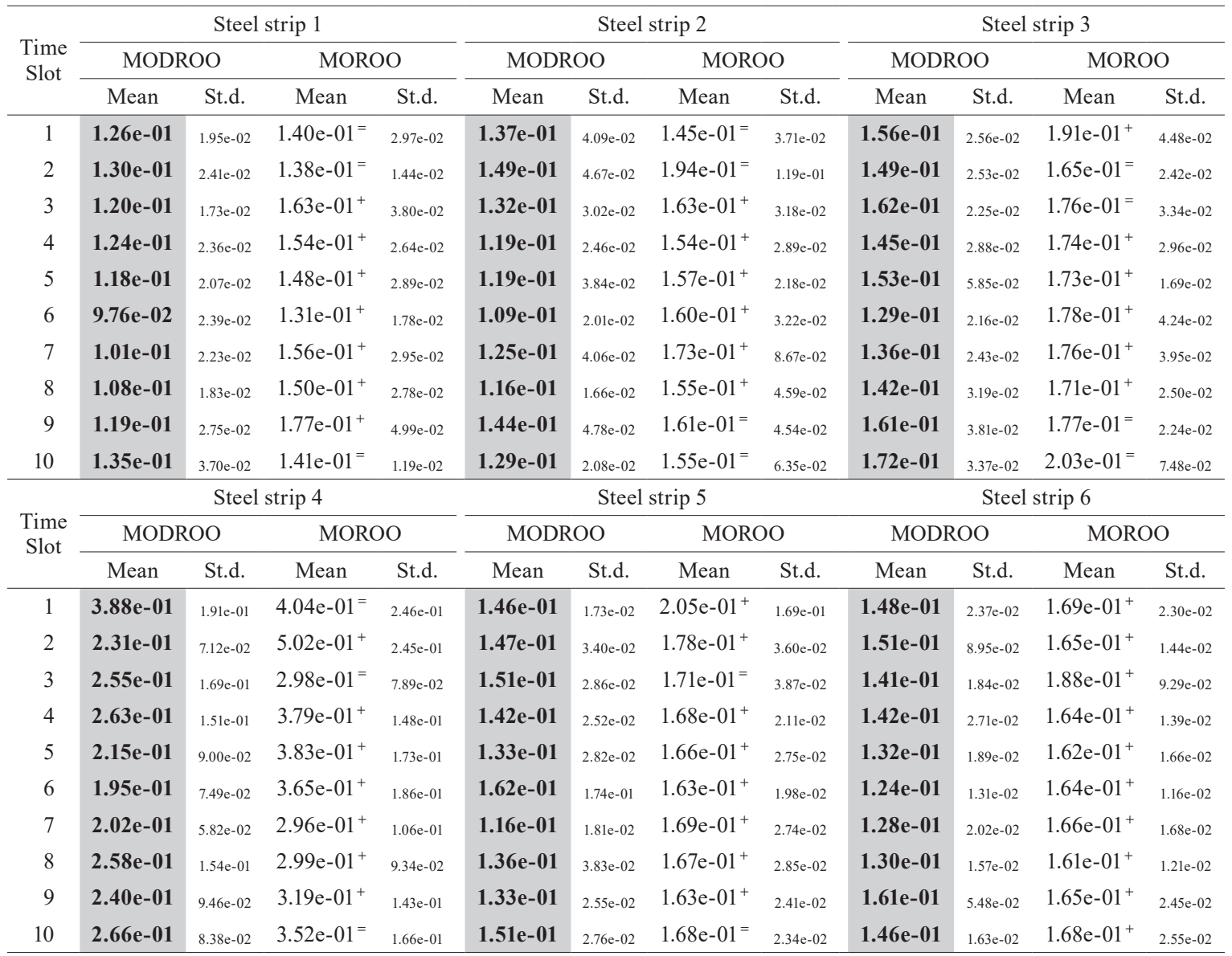

Table 8. Computational results of IGD for the first three strips obtained by MOROO and MODROO for Case 2.

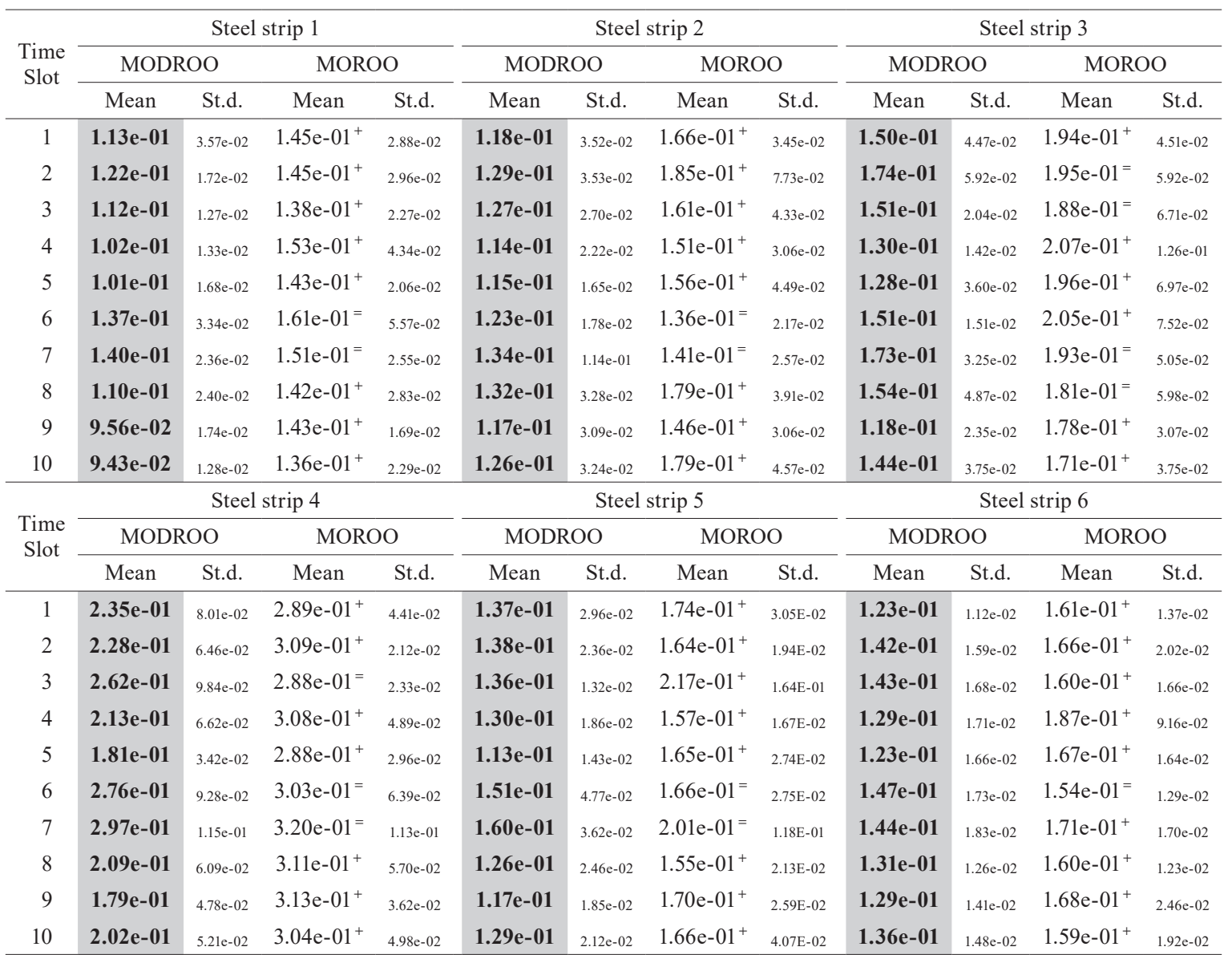


Table 9. Computational results of IGD for the first three strips obtained by MOROO and MODROO for Case 3 .

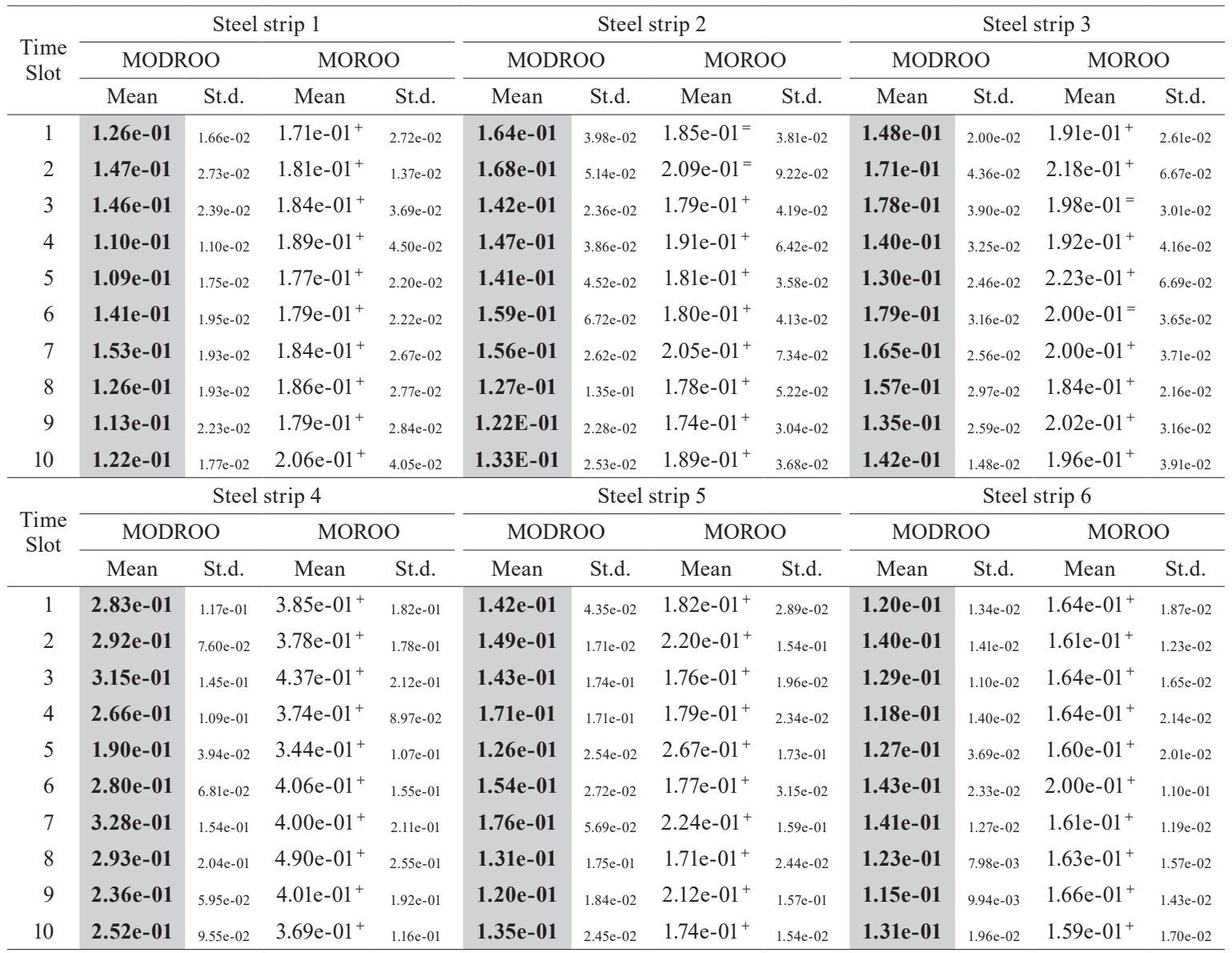

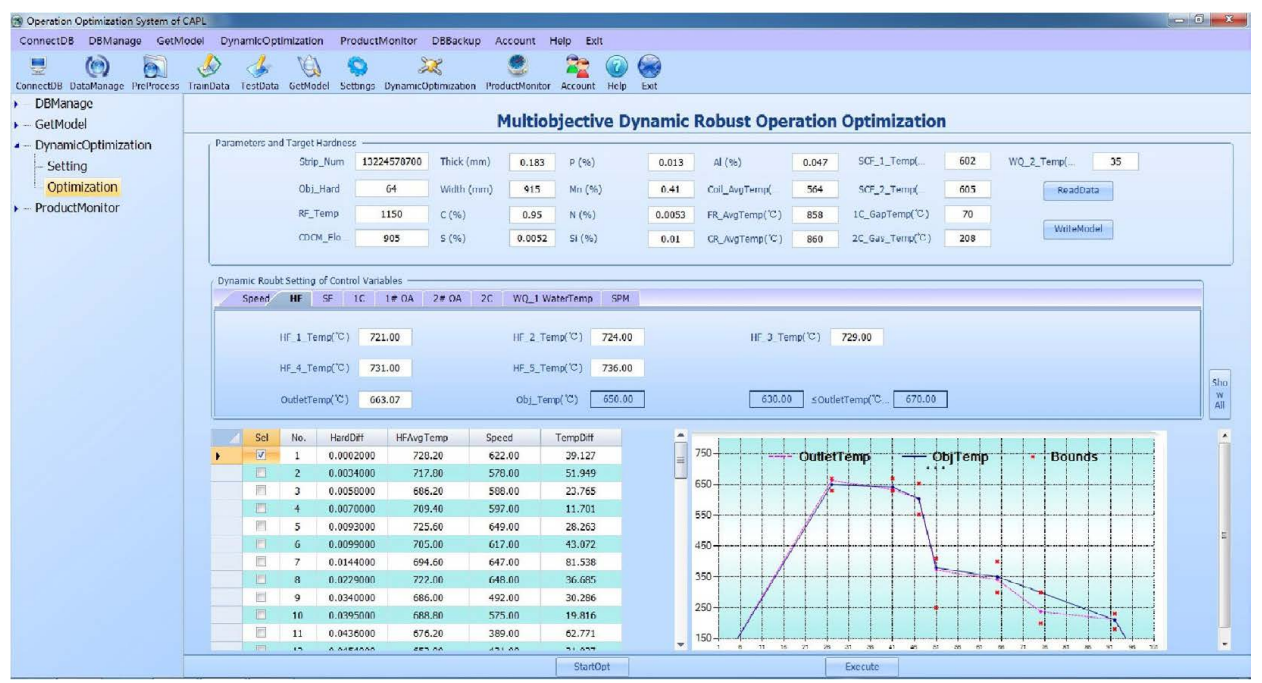

Fig. 8. Application system of MODROO-CAPL in a major iron and steel enterprise. (Online version in color.)

tional MOROO in three different cases are presented in Table 7, Tables 8 and 9, respectively. From the results, it can be seen that MODROO can obtain better results for all time slots, and the IGD results of MODROO are significantly better than those of traditional MOROO for most time slots. For certain time slots in each case, the performance difference between the two models is not significant. The main reason is that the predicted disturbance ranges of MODROO in these time slots are close to the maximum range of MOROO. However, the number of time slots for which the two models have no significant performance dif- ference decreases from 16 for Case 1, 14 for Case 2) to 4 for Case 3. This means that the problem becomes more difficult as the increase of the number of disturbances and that the advantage of MODROO model will be more significant with comparison to traditional MOROO model for difficult problems.

\subsection{Application of MODROO-CAPL in Practical Pro- duction}

For the obtained non-dominated solutions in the EXA by the improved NSGA-II, the TOPSIS method ${ }^{35)}$ is adopted to 
select one appropriate solution and then the selected solution will be applied in each time slot in practical production. During production in each time slot, we will also monitor the real disturbance range. Once it exceeds the predicted range, we will terminate current rolling time horizon and start a new one; otherwise, the production continues with the selected setting of variables until current rolling time horizon terminates. Based on the above application framework, a decision support system for the dynamic robust operation optimization of CAPL is developed, as shown in Fig. 8, and tested in one of the major iron and steel enterprise in China.

\section{Conclusions}

Different from the traditional MOROO modeling method, this paper developed a new MODROO modeling method in which the dynamic change of disturbance range in practical production is considered and analyzed by a datadriven method. The main feature of the MODROO is that a time series prediction model based on LSSVR is used to predict the maximum disturbance range for each potential variable in the next time slot within the production time horizon, which in turn makes the robustness of solution be dynamically adjusted along the time slots. To efficiently solve the MODROO model, an improved NSGA-II algorithm is proposed using a new crowding distance metric based on the solution distribution in the objective space. Experimental results on benchmark problems show that the proposed improved NSGA-II algorithm is superior to some of the powerful MOEAs in the literature. Three cases of different number of disturbance range changes in CAPL are used to verify the proposed MODROO model, and the computational results show that the MODROO model is significantly better than the traditional MOROO model for most strips. Besides, the results illustrate that the advantage of MODROO becomes more significant as the increase of the number of disturbance changes.

\section{Acknowledgement}

This research is partly supported by National Key Research and Development Program of China (2018YFB1700404), the Fund for the National Natural Science Foundation of China (61573086), the Major Program of National Natural Science Foundation of China (71790614), the Fund for Innovative Research Groups of the National Natural Science Foundation of China (71621061), the Major International Joint Research Project of the National Natural Science Foundation of China (71520107004), and the 111 Project (B16009).

\section{REFERENCES}

1) C. Guo, Y. W. Zhang, X. You, X. Chen and Y. Zhang: IEEE Int. Conf. on Automation and Logistics, IEEE, Piscataway, NJ, (2009), 602.

2) Y. Y. Zhang, Y. L. Jin, W. H. Cao, Z. Z. Li and Y. Yuan: Proc. 37th Chinese Control Conf., IEEE, Piscataway, NJ, (2018), 1887.

3) D. Mazumdar, P. Dhandapani and R. Sarvanakumar: ISIJ Int., 57 (2017), 286.

4) Y. H. Mu, B. Y. Wang, J. Zhou, Y. Kang and X. T. Li: ISIJ Int., 57 (2017), 1442.

5) H. Helle, M. Helle, F. Pettersson and H. Saxén: ISIJ Int., 50 (2010), 1380.

6) X. D. Gao, B. Z. Chen, X. R. He, T. Qiu, J. C. Li, C. M. Wang and L. J. Zhang: Comput. Chem. Eng., 32 (2008), 2801.

7) X. P. Wang and L. X. Tang: Ind. Eng. Chem. Res., 52 (2013), 14415.

8) T. Jia, Z. Y. Liu, H. F. Hu and G. D. Wang: ISIJ Int., 51 (2011), 1468 .

9) L. Chen and X. P. Wang: Ind. Eng. Chem. Res., 53 (2014), 11393.

10) Q. Xia, X. P. Wang and L. X. Tang: ISIJ Int., 56 (2016), 2006.

11) Q. Xia, X. P. Wang and L. X. Tang: Soft Comput., 23 (2019), 9551.

12) H. G. Beyer and B. Sendhoff: Comput. Methods Appl. Mech. Eng., 196 (2007), 3190.

13) Y. Hong and X. P. Wang: 26th Chinese Control and Decision Conf., IEEE, Piscataway, NJ, (2014), 1365.

14) A. Leiras, S. Hamacher and A. Elkamel: Eng. Optim., 42 (2010), 1119.

15) M. S. Govatsmark and S. Skogestad: Ind. Eng. Chem. Res., 44 (2005), 2207.

16) E. Yasari, M. R. Pishvaie, F. Khorasheh, K. Salahshoor and R. Kharrat: J. Pet. Sci. Eng., 109 (2013), 1.

17) K. Deb and H. Gupta: Evol. Comput., 14 (2006), 463.

18) J. A. K. Suykens and J. Vandewalle: Neural Process. Lett., 9 (1999), 293.

19) K. Deb, A. Pratap, S. Agarwal and T. Meyarivan: IEEE Trans. Evol. Comput., 6 (2002), 182.

20) K. Deb and R. B. Agrawal: Complex Syst., 9 (1995), 115.

21) A. J. Nebro, F. Luna, E. Alba, B. Dorronsoro, J. J. Durillo and A Beham: IEEE Trans. Evol. Comput., 12 (2008), 439.

22) H. Li and Q. F. Zhang: IEEE Trans. Evol. Comput., 13 (2009), 284.

23 L. X. Tang and X. P. Wang: IEEE Trans. Evol. Comput., 17 (2013), 20.

24) L. X. Tang, Y. Dong and J. Y. Liu: IEEE Trans. Evol. Comput., 19 (2015), 560

25) L. X. Tang, Y. Zhao and J. Y. Liu: IEEE Trans. Evol. Comput., 18 (2014), 209.

26) K. Li, Q. F. Zhang, S. Kwong, M. Li and R. Wang: IEEE Trans. Evol. Comput., 18 (2014), No. 6, 909.

27) X. P. Wang and L. X. Tang: Inf. Sci., 348 (2016), 124

$28)$ L. X. Tang, X. P. Wang and Z. M. Dong: IEEE Trans. Cybern., 9 (2019), 3571.

29) X. P. Wang, Z. M. Dong, L. X. Tang: IEEE Trans. Syst., Man., Cyber. Syst., in press. https://doi.org/10.1109/TSMC.2018.2875043

30) N. Agrawal, G. P. Rangaiah, A. K. Ray and S. K. Gupta: Ind. Eng. Chem. Res., 45 (2006), 3182

31) E. Zitzler, K. Deb and L. Thiele: Evol. Comput., 8 (2000), 173.

32) Q. F. Zhang, A. M. Zhou, S. Zhao, P. N. Suganthan, W. Liu and S. Tiwari: Technical Report CES-487, The School of Computer Science and Electronic Engineering, University of Essex, Essex, U.K., (2008).

$33)$ P. A. N. Bosman and D. Thierens: IEEE Trans. Evol. Comput., 7 (2003), 174.

34) S. Kukkonen and J. Lampinen: IEEE Congr. on Evolutionary Computation, IEEE, Piscataway, NJ, (2005), 443.

35) S. J. Chen and C. L. Hwang: Fuzzy Multiple Attribute Decision Making, Springer, Berlin, Heidelberg, (1992), 289. 